\title{
Vom Wert der Familienanamnese beim CRC
}

\author{
Einer von 14 Hausarztpatienten im Alter zwischen 40 und 54 Jahren hat zumindest einen Verwandten \\ 1. Grades mit einem kolorektalen Karzinom (CRC). Die Familienanamnese lohnt sich also unbedingt!
}

_ Im Alter von 55 Jahren haben GKVPatienten Anspruch auf eine Vorsorgekoloskopie. Für Personen mit familiärer Belastung für ein kolorektales Karzinom (CRC) wird die erste Untersuchung allerdings bereits zehn Jahre früher empfohlen. Nun wurde in hessischen Hausarztpraxen untersucht, wie häufig eine positive Familienanamnese bei 40bis 54-jährigen Patienten ist.

Per Fragebogen wurde eruiert, ob es mindestens einen Verwandten 1. Grades mit CRC gibt, ob diese Person ihre Diagnose vor dem 50. Lebensjahr erhalten hat, und ob es insgesamt drei oder mehr Verwandte mit CRC, Magen-, Zervix-,
Ovarial-, Blasen- oder Nierenkarzinomgibt. 21 Praxen mit 12.184 geeigneten Patienten wurden eingeschlossen. Am Ende stimmten 6.723 Patienten einer Befragung zu.

7,2\% der Teilnehmer berichteten über mindestens einen Verwandten 1. Grades mit einem CRC. Bei 1,2\% der Befragten trat dieses Karzinom bei dem Verwandten vor dem 50. Lebensjahr auf, weitere 2,6\% hatten einen Verwandten 1. Grades, bei dem vor dem 50. Lebensjahr ein kolorektaler Polyp entfernt worden war. Drei oder mehr Verwandte mit den oben genannten Karzinomerkrankungen hatten 2,1\% der Befragten.
- Plath J, Siebenhofer A, Konél et al. Frequency of a positive family history of colorectal cancer in general practice: a crosssectional study. Family Practice. 2017;34:30-5

\section{KOMMENTAR}

Die Daten zeigen, dass man unter 14 befragten Patienten zwischen 40 und 54 Jahren einen findet, der einen Verwandten 1. Grades mit einem CRC hat - eine durchaus respektable Quote. Mit diesem einfachen Vor-Screening kann man sicher die Akzeptanz der Vorsorge-Koloskopie erhöhen und die "richtigen" $\mathrm{Pa}$ tienten mit erhöhtem Risiko überweisen. Hausärzt sollten diesen Sachverhalt mit ihren Patienten besprechen.

Prof. Dr. med. H. S. FüeßI

\section{Hier steht eine Anzeige.}

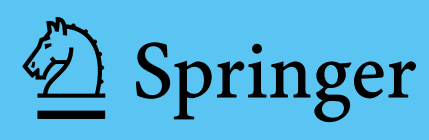

